\title{
Optical Fibers Enter a New Space-Time Era
}

\author{
K. Krupa ${ }^{1,2}$, A. Tonello ${ }^{1}$, R. Dupiol ${ }^{2}$, A. Bendahmane ${ }^{2}$, B. Mohamed Shalaby ${ }^{1,3}$, M. Fabert ${ }^{1}$, \\ A. Barthélémy ${ }^{1}$, G. Millot ${ }^{2}$, S. Wabnitz ${ }^{4}$, and V. Couderc ${ }^{1}$ \\ ${ }^{1}$ Université de Limoges, XLIM, UMR CNRS 7252, 123 avenue A. Thomas, 87060 Limoges, France \\ ${ }^{2}$ Université de Bourgogne Franche-Comté, ICB, UMR CNRS 6303, 9 avenue A. Savary, 21078 Dijon, France \\ ${ }^{3}$ Tanta University, Faculty of Science, Physics Department, Tanta, Egypt \\ ${ }^{4}$ Università di Brescia, Dipartimento di Ingegneria dell'Informazione, and INO-CNR, via Branze 38, 25123 Brescia, Italy
} katarzyna.krupa@unilim.fr

\begin{abstract}
We show experimentally a new type of parametric instability associated with the original phenomenon of beam self-cleaning in multimode fibers. Our experimental results are in good agreement with numerical solutions of the Gross-Pitaevskii equation.

OCIS codes: (060.4370) Nonlinear optics, fibers; (190.4410) Nonlinear optics, parametric processes
\end{abstract}

\section{Introduction}

Single-mode optical fibers have revolutionized the telecommunication industry. They have proved to be very successful for studying the purely temporal dynamics of nonlinear optical waves. However, multimode fibers are today experiencing a strong renewed interest for spatial-division multiplexing [1], and offer new perspectives thanks to their complex mixing of temporal and spatial effects [2-5]. Graded-index multimode fibers (GRIN MMFs) have recently received particular attention, as they enabled the first remarkable observation of multimode femtosecond solitons in the anomalous dispersion regime, and their associated resonant dispersive waves [2-5].

In this work, we introduce a new regime for the operation of MMFs, by experimentally and numerically exploring the spatiotemporal dynamics of sub-nanosecond pulses, propagating in the normal dispersion regime of GRIN MMFs. We used power-chip Nd:YAG lasers delivering pulses from 500 to $900 \mathrm{ps}$ at $1064 \mathrm{~nm}$. A first study deals with an optical fiber supporting only five modes at the excitation wavelength (and two modes at $1550 \mathrm{~nm}$ ). In such weakly multimode regime, the spatiotemporal dynamics can be well understood by a detailed analysis of both phase-matching conditions and overlap integrals of the various intermodal four-wave mixing processes. This permits to predict both wavelengths and modal content of the main parametric spectral peaks [6]. We show that such a weakly multimode fiber allows for an exceptionally wide frequency shift $f_{l} \cong 200 \mathrm{THz}$ for the first pair of parametric sidebands. However, a comprehensive treatment of the intermodal four-wave mixing requires taking into account the multitude of possible nonlinear interactions between the individual modes. Such complex analysis requires significant computational effort, which considerably increases as the number of excited modes grows larger.

If tens or hundreds of guided modes are simultaneously excited in a highly MMF, the approach based on individual modes quickly becomes inappropriate, and a collective approach based on the direct study of the dynamics of the total field is strongly preferable [7]. Beam propagation along a GRIN MMF is characterized by the natural periodic spatial oscillation of the optical field. This creates, via the Kerr effect, a dynamic (or self-induced) longitudinal refractive index grating with submillimeter period. The associated quasi-phase-matching of four wave mixing is responsible for activating a new type of geometric parametric instability, generating an intense array of sidebands covering the ultrawide spectral range from the near ultraviolet to the near infrared. First-order sidebands are characterized by their very large frequency shift $f_{1}=123.5 \mathrm{THz}[8]$. The sideband frequencies are not equidistant, as their shift from the pump is proportional to the square root of their order n, namely $f_{n} \cong \sqrt{n} f_{1}$.

In addition, we found that the Kerr nonlinearity in GRIN MMF permits the striking effect of beam self-cleaning, which is induced even in the absence of any wavelength conversion process. The input laser beam emerges from the MMF at low powers (linear regime) as a pattern of speckles, owing to interference among the very large number of excited modes. On the other hand, at high powers the output beam exhibits a sudden increase of its own spatial coherence: the laser beam emerges from the fiber with a nearly Gaussian shape, similar to that of a fundamental mode. Another remarkable result of our observations is that the phenomenon of spatial beam self-organization is very robust against strong external mechanical perturbations.

\section{A few typical observations}

Figures 1 and 2 present typical examples of experimental spectra recorded at the output of a weakly and highly multimode fiber, respectively. Each example shows the appearance of a series of parametric sidebands, nonuniformly distributed over a wide spectral range. We observe a strong energy conversion into the visible of the pump wavelength at $1064 \mathrm{~nm}$. For example, a green wave at $513 \mathrm{~nm}$ is generated with a peak power of $1.2 \mathrm{~kW}$ (case 
of Fig.1). The yellow-green and red parametric lines at $553 \mathrm{~nm}$ and $623 \mathrm{~nm}$, respectively, are sufficiently intense to generate their own cascade of self-stimulated Raman Stokes waves.

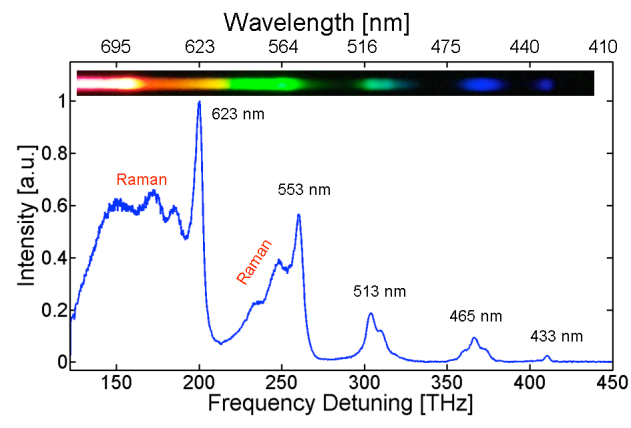

Fig. 1. Experimental spectrum obtained at the output of a $10 \mathrm{~m}$-long weakly multimode fiber with $32 \mathrm{~kW}$ of input peak power.

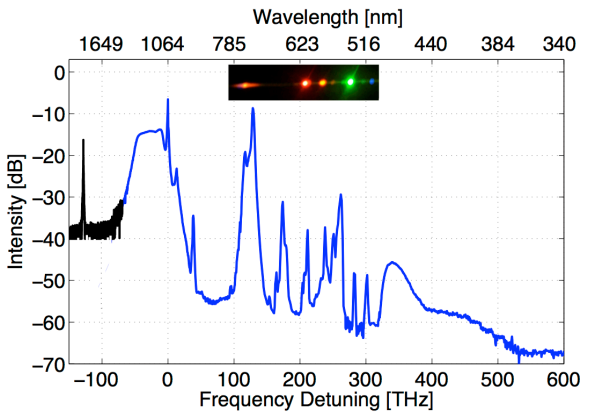

Fig. 2. Experimental spectrum obtained at the output of a $6 \mathrm{~m}-$ long highly multimode fiber with $50 \mathrm{~kW}$ of input peak power.

Figure 3 shows the spatial beam profiles measured at the output of the $6 \mathrm{~m}$-long highly multimode fiber. The initially incoherent spatial structure (speckle) becomes coherent at high powers. This remarkable effect of beam selfcleaning is achieved at all parametric spectral lines (Fig.3c-f). Note that a low-power incoherent background remains at the pump wavelength (Fig.3b), which is sufficient to generate the observed parametric sidebands. Our experimental results are in good quantitative agreement with numerical solutions of the $(3+1) \mathrm{D}$ spatiotemporal nonlinear Schrödinger equation, also known as the Gross-Pitaevskii equation for the Bose-Einstein condensation.
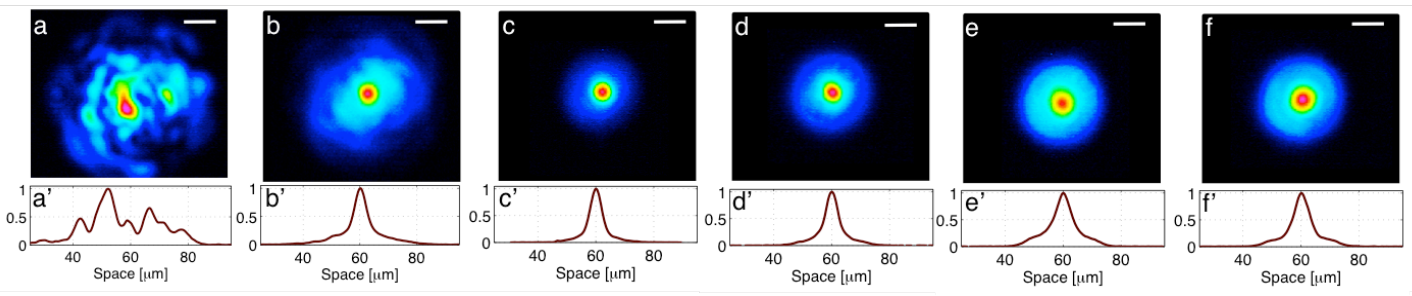

Fig. 3. Spatial profiles of the pump at $1064 \mathrm{~nm}$ with an input peak power of $0.06 \mathrm{~kW}\left(\mathrm{a}, \mathrm{a}^{\prime}\right)$ and $50 \mathrm{~kW}\left(\mathrm{~b}, \mathrm{~b}^{\prime}\right)$; same for the anti-Stokes lines at $750 \mathrm{~nm}\left(\mathrm{c}, \mathrm{c}^{\prime}\right), 650 \mathrm{~nm}\left(\mathrm{~d}, \mathrm{~d}^{\prime}\right), 600 \mathrm{~nm}\left(\mathrm{e}, \mathrm{e}^{\prime}\right)$ and $550 \mathrm{~nm}\left(\mathrm{f}, \mathrm{f}^{\prime}\right)$ at input peak power $50 \mathrm{~kW}$. Scale bar $=10 \mu \mathrm{m}$.

\section{Conclusion}

We observed the generation of an ultrawideband array of spectral sidebands and the spatial beam self-cleaning, induced by Kerr effect in the normal dispersion regime of GRIN MMFs. Our results provide a significant contribution to the emerging field of the complex spatiotemporal systems. They open the way for a plethora of promising innovative applications, such as: the development of new powerful and broadband multipurpose fibered sources, the control of the spatial coherence of laser beams, the generation of intense supercontinuum, to cite a few.

\section{References}

[1] D.J. Richardson, J.M. Fini and L.E. Nelson, “Space division multiplexing in optical fibers,” Nat. Photonics 7, 354-362 (2013).

[2] L. G. Wright, D. N. Christodoulides and F. W. Wise, "Controllable spatiotemporal nonlinear effects in multimode fibres," Nat. Photonics 9 , 306-310 (2015).

[3] W.H. Renninger and F.W. Wise, “Optical solitons in graded-index multimode fibres,” Nat. Commun. 4, 1719 (2013).

[4] L. G. Wright, S. Wabnitz, D.N. Chistodoulides and F.W. Wise, "Ultrabroadband dispersive radiation by spatiotemporal oscillation of multimode waves," Phys. Rev. Lett. 115, 223902 (2015).

[5] A. Picozzi, G. Millot and S. Wabnitz, "Nonlinear virtues of multimode fibre,” Nat. Photonics 9, 289-291 (2015).

[6] E. Nazemosadat, H. Pourbeyram and A. Mafi, "Phase matching for spontaneous frequency conversion via four-wave mixing in graded-index multimode optical fibers," J. Opt. Soc. Am. B 33, 144-150 (2016).

[7] S. Longhi, "Modulational instability and space-time dynamics in nonlinear parabolic-index optical fibers," Opt. Lett. 28, $2363-2365$ (2003).

[8] K. Krupa, A. Tonello, B. M. Shalaby, A. Barthélémy, V. Couderc, A. Bendahmane, G. Millot and S. Wabnitz, "Observation of geometric parametric instability sidebands by the periodic spatial self-imaging of multimode waves," to appear in Phys. Rev. Lett. 